THEOREM 4. If the boundary $\Gamma$ of the plane bounded connected and simply connected domain $\gamma$ contains an indecomposable continuum $D$, there is a prime end of $\gamma$ which contains $D$.

Here, as in the development of Theorem 2, for each value of $j$ the set $\Gamma=\sum \Gamma_{j i}$. Consequently $\sum \Gamma_{j i} \supset D$. If for each of these $\overline{c\left(\Gamma_{j i}\right) \cdot D} \supset D$, then the set $\sum \Gamma_{j i} \cdot D$ is nowhere dense in $D$ and $\left[\Gamma_{j i}\right]$ does not cover $D$. But as none of $\left[\Gamma_{j i}\right]$ can have $\overline{c\left(\Gamma_{j i}\right) \cdot D}$ $\$ D$ unless $D \cdot c\left(\Gamma_{j i}\right)=0$, in view of Lemma 4 , there must for every value of $j$ be one of $\left[\Gamma_{j i}\right]$ which contains $D$. The proof now follows lines almost identical with those of Theorem 2 .

NORTHWESTERN UNIVERSITY

\title{
PROJECTIVE DIFFERENTIAL GEOMETRY OF CURVES
}

BY L. R. WILCOX

In a fundamental paper* on the projective differential geometry of curves, L. Berzolari obtained canonical expansions representing a curve $C$ immersed in a linear space $S_{n}$ in a neighborhood of one of its points $P_{0}$. The vertices of the coordinate simplex yielding Berzolari's canonical form are covariantly related to the curve, while the unit point may be any point of the rational normal curve $\Gamma$ which osculates $C$ at $P_{0}$. It is the purpose of the present paper to define a covariant point on $\Gamma$ which can be chosen as a unit point so as to produce final canonicalization of the power series expansions of Berzolari.

It will be observed that the usual methods of defining a point on $\Gamma$ for the cases $n=2$ and $n=3$ depend on configurations $\dagger$ that do not possess suitable analogs in $n$-space. Hence it appeared for some time that the problem called for different procedures in spaces of different dimensionality. Special devices

* L. Berzolari, Sugli invarianti differenziali proiettivi delle curve di un iperspazio, Annali di Matematica, (2), vol. 26 (1897), pp. 1-58.

$\dagger$ E. P. Lane, Projective Differential Geometry of Curves and Surfaces, pp. 12-27. 
were found by S. B. Murray and the author* for the spaces $S_{4}$ and $S_{5}$; however, like the methods used in the plane and in $S_{3}$ these seem not to admit generalization. It is to be shown here that, with the help of a suitably chosen linear complex, the general problem for $n>3$ may be solved.

Local power series expansions representing an analytic curve $C$ immersed in a linear space $S_{n}$ of $n$ dimensions $(n>3)$ in a neighborhood of an ordinary point $P_{0}$ may be written $\dagger$ in the form,

$$
\begin{aligned}
& x_{0}=1, \\
& x_{i}=x_{1}^{i}+a_{i} x_{1}^{n+3}+b_{i} x_{1}^{n+4}+\cdots, \quad(i=2, \cdots, n),
\end{aligned}
$$

wherein $x_{0}, \cdots, x_{n}$ are homogeneous projective point coordinates, and the coefficients $a_{i}, b_{i}$, etc. are complex numbers, $a_{n-1}$ being zero and $a_{n}$ different from zero. The equations of the osculating rational normal curve $\Gamma$ of $C$ at $P_{0}$ are

$$
x_{i}=x_{1}{ }^{i}, \quad(i=0, \cdots, n) .
$$

The vertices of the coordinate simplex will be denoted by $P_{0}, \cdots, P_{n}$, where $P_{i}$ is the point for which

$$
x_{i}=1, \quad x_{j}=0, \quad(j=0, \cdots, n ; j \neq i) .
$$

The point $P_{n}$ is the intersection that is distinct from $P_{0}$ of the curve $\Gamma$ and the principal hyperplane $\ddagger$ of $C$ and $\Gamma$; the vertex $P_{i},(i=1, \cdots, n-1)$, is the intersection of the osculating space $S_{n-i}$ of $\Gamma$ at $P_{n}$ and the osculating space $S_{i}$ of $C$ at $P_{0}$. The unit point $U(1, \cdots, 1)$ is any point on $\Gamma$ distinct from the points $P_{0}$ and $P_{n}$.

Homogeneous line coordinates $p_{i j}$ of the line joining points $X\left(x_{0}, \cdots, x_{n}\right)$ and $Y\left(y_{0}, \cdots, y_{n}\right)$ will be defined by

* See Murray, Curves in Four-Dimensional Space, Chicago master's dissertation, 1934, and Wilcox, Curves in Five-Dimensional Space, Chicago master's dissertation, 1933.

† Berzolari, loc. cit., p. 2. We shall say that $P_{0}$ is an ordinary point of $C$ in case (1) $C$ is not hyperosculated at $P_{0}$ by any of its linear osculants or by its osculating rational normal curve $\Gamma$, and (2) $C$ and $\Gamma$ have at $P_{0}$ a principal plane not contained in their osculating hyperplane at $P_{0}$. For the definition of principal plane see Berzolari, loc. cit., p. 18.

$\ddagger$ Berzolari, loc. cit., p. 19. 


$$
p_{i j}=x_{i} y_{j}-x_{j} y_{i}, \quad(i, j=0, \cdots, n ; i<j) .
$$

The coordinates of the line $l_{h k}$ joining $P_{h}$ and $P_{k}(h<k)$ are given by

$$
p_{i j}=\left\{\begin{array}{l}
1, \text { when } i=h \text { and } j=k, \\
0, \text { when } i \neq h \text { or } j \neq k .
\end{array}\right.
$$

In the totality of linear complexes in the ambient space $S_{n}$ there is a two-parameter family containing all lines $l_{h k}$ except $l_{0, n}, l_{1, n-1}$, and $l_{3, n}$. The equation of this family is

$$
\lambda p_{0, n}+\mu p_{1, n-1}+\nu p_{3, n}=0,
$$

wherein $\lambda, \mu, \nu$ are homogeneous parameters. In the family (2) there is a unique complex having $(n+3)$-line contact with the tangent developable of the curve $C$ at the line $l_{0,1}$. With the help of expansions (1) its equation is found to be

$$
\begin{aligned}
(n-2)(n-3) p_{0, n}-n(n-3) p_{1, n-1} \\
-(n-2)(n+3) a_{n} p_{3, n}=0 .
\end{aligned}
$$

The locus of all lines of the complex (3) through the point $P_{n}$ is a hyperplane $\pi$ whose equation is

$$
(n-3) x_{0}-(n+3) a_{n} x_{3}=0 .
$$

If we demand that the unit point $U$ shall lie in this hyperplane, we have

$$
a_{n}=\frac{n-3}{n+3}
$$

hence we obtain the following result.

$A n$ analytic curve $C$ immersed in a linear space of $n$ dimensions may be represented in a neighborhood of one of its ordinary points $P_{0}$ by local power series expansions of the form (1), in which $a_{n}=(n-3) /(n+3)$. For this canonical form the unit point is one of the intersections distinct from $P_{n}$ of the hyperplane $\pi$ with the osculating rational normal curve of $C$ at $P_{0}$.

University of Chicago 